\title{
Melphalan Flufenamide
}

National Cancer Institute

\section{Source}

National Cancer Institute. Melphalan Flufenamide. NCI Thesaurus. Code C107680.

A melphalan prodrug in which the alkylating agent melphalan is bound to flufenamide, with potential antineoplastic and anti-angiogenic activities. Upon administration, the dipeptide bond in the melphalan-flufenamide compound is hydrolyzed by peptidases, which are overexpressed by certain cancer cells. This results in the specific release and accumulation of the active metabolite melphalan in cancer cells. Melphalan alkylates DNA at the N7 position of guanine residues and induces DNA intra- and inter-strand crosslinkages. This results in the inhibition of DNA and RNA synthesis and the induction of apoptosis, thereby inhibiting tumor cell proliferation. The administration of the melphalan-flufenamide prodrug allows for enhanced efficacy and reduced toxicity compared to melphalan alone. 